\title{
REMARKS ON A BOUNDARY VALUE PROBLEM IN BANACH SPACES ON THE HALF-LINE
}

\author{
WOJCIECH GRĄZIEWICZ \\ Department of Technical Physics and Applied Mathematics \\ Gdańsk University of Technology \\ Narutowicza 11/12, 80-952 Gdańsk \\ E-mail: graz@mif.pg.gda.pl
}

\begin{abstract}
In this paper we investigate the linear initial value problem in Banach spaces. In order to obtain existence results the Fredholm operator technique is used.
\end{abstract}

In the paper [3] the authors consider the problem of the existence of solutions in Sobolev space $H^{1}\left(\overline{\mathbb{R}}_{+}, \mathbb{R}^{N}\right)$ for the ODE system

$$
\left\{\begin{array}{l}
\dot{u}+F(t, u)=f(t), \quad t \in \overline{\mathbb{R}}_{+}=[0, \infty), \\
u_{1}(0)=\zeta,
\end{array}\right.
$$

where $F: \overline{\mathbb{R}}_{+} \times \mathbb{R}^{N} \longrightarrow \mathbb{R}^{N}$ is a $C^{1}$ mapping such that $F(t, 0)=0$ for all $t \in \overline{\mathbb{R}}_{+}$, and $u_{1}$ is the component of $u$ along the first factor of a given splitting $\mathbb{R}^{N}=X_{1} \oplus X_{2}$. Both $f \in L^{2}\left(\mathbb{R}_{+}, \mathbb{R}^{N}\right)$ and $\zeta \in X_{1}$ are given.

In the case $X_{1}=\mathbb{R}^{N}$ and $X_{2}=\{0\}$ the above problem is the classical initial value problem. Our aim is to generalize this problem to the infinite-dimensional case. In this work we investigate only the linear equation.

Let us fix the notation.

Let $(E,\|\cdot\|)$ be a real separable Banach space. Denote by $\mathcal{L}(E)$ the space of linear bounded operators on $E$ and by $\mathcal{L}_{c}(E)$ the subspace of $\mathcal{L}(E)$ consisting of compact operators.

For a measurable function $u:(a, b) \longrightarrow E,-\infty \leq a<b \leq \infty$, the integral $\int_{a}^{b} u(t) d t$ means the integral in Bochner sense. We recall that a measurable function $u(\cdot)$ is (Bochner) integrable on $(a, b)$ if and only if the real function $\|u(\cdot)\|$ is (Lebesgue) inte-

2000 Mathematics Subject Classification: Primary: 34B40; Secondary: 46E35.

Key words and phrases: ordinary differential equations; Sobolev space; boundary value problem; Fredholm operator.

Supported by Grant KBN No. 1 P03A 04229.

The paper is in final form and no version of it will be published elsewhere. 
grable on $(a, b)$. The vector space $L^{2}=L^{2}\left(\overline{\mathbb{R}}_{+}, E\right)$ consists of all functions $u: \overline{\mathbb{R}}_{+} \longrightarrow E$ such that $\|u\| \in L^{2}\left(\overline{\mathbb{R}}_{+}, \mathbb{R}\right)$ with the norm $\|u\|_{2}=\left(\int_{0}^{\infty}\|u(t)\|^{2} d t\right)^{1 / 2}$. We denote by $H^{1}=H^{1}\left(\overline{\mathbb{R}}_{+}, E\right)$ the Sobolev space of such functions $u: \overline{\mathbb{R}}_{+} \longrightarrow E$ that $u, \dot{u} \in L^{2}$. The space $H^{1}$ is equipped with the norm $\|u\|_{H^{1}}=\left(\|u\|_{2}^{2}+\|\dot{u}\|_{2}^{2}\right)^{1 / 2}$. We recall that the derivative $\dot{u}$ means the derivative in the distributional sense.

It can be shown that if $u \in H^{1}$ and $\widetilde{u}$ is a continuous function such that $u=\widetilde{u}$ a.e. on $\overline{\mathbb{R}}_{+}$, then $\lim _{t \rightarrow \infty}\|\widetilde{u}(t)\|=0$.

The main problem. Let $A \in \mathcal{L}(E)$ be such that $\sigma(A) \cap \mathbb{R} i=\emptyset$, where $\sigma(A)$ denotes the spectrum of the operator $A$. Then $A$ gives the standard unique decomposition $E=$ $X_{+} \oplus X_{-}$, where the subspaces $X_{+}$and $X_{-}$are invariant with respect to $A$. The operators $A_{+}:=\left.A\right|_{X_{+}}, A_{-}:=\left.A\right|_{X_{-}}$have their spectra contained in $\mathbb{C}_{+}=\{\lambda \in \mathbb{C}: \operatorname{Re}(\lambda)>0\}$ and $\mathbb{C}_{-}$, respectively. Every function $u: \overline{\mathbb{R}}_{+} \longrightarrow E$ has a unique decomposition of the form $u=u_{+}+u_{-}$, where $u_{ \pm}: \overline{\mathbb{R}}_{+} \longrightarrow X_{ \pm}$.

Proposition 1. Under the above conditions the linear mapping

$$
H^{1} \ni u \longmapsto\left(\dot{u}+A u, u_{+}(0)\right) \in L^{2} \times X_{+}
$$

is an isomorphism.

Proof. Fix $\zeta_{+} \in X_{+}, f \in L^{2}$. We will show that the problem

$$
\left\{\begin{array}{l}
\dot{u}+A u=f \\
u_{+}(0)=\zeta_{+}
\end{array}\right.
$$

has a unique solution $u \in H^{1}$. The above problem is equivalent to the conjunction of two problems:

$$
\left\{\begin{array}{l}
\dot{u}_{+}+A_{+} u_{+}=f_{+} \\
u_{+}(0)=\zeta_{+}
\end{array} \quad \text { and } \quad \dot{u}_{-}+A_{-} u_{-}=f_{-} .\right.
$$

The first is a Cauchy problem in the Banach space $X_{+}$and has a unique solution given by the formula

$$
u_{+}(t)=e^{-t A_{+}} \zeta_{+}+\int_{0}^{t} e^{-(t-s) A_{+}} f_{+}(s) d s .
$$

We will show that $u_{+} \in H^{1}$. First we consider the function

$$
\overline{\mathbb{R}}_{+} \ni t \longmapsto e^{-t A_{+}} \zeta_{+} \in X_{+} .
$$

Because $\sigma\left(-\left.t A\right|_{X_{+}}\right)=\sigma\left(-t A_{+}\right) \subset \mathbb{C}_{-}$, there are $\alpha>0$ and $M>0$ such that $\left\|e^{-t A_{+}} \zeta_{+}\right\| \leq\left\|e^{-t A_{+}}\right\| \cdot\left\|\zeta_{+}\right\| \leq M e^{-\alpha t} \cdot\left\|\zeta_{+}\right\|$. Therefore

$$
\int_{0}^{\infty}\left\|e^{-t A_{+}} \zeta_{+}\right\|^{2} d t \leq \int_{0}^{\infty}\left(M e^{-\alpha t} \cdot\left\|\zeta_{+}\right\|\right)^{2} d t<\infty
$$

and it follows that the function (4) belongs to $L^{2}$. In order to estimate the second component of the function defined in (3) we will use the Young inequality:

$$
\|g * f\|_{r} \leq\|g\|_{p} \cdot\|f\|_{q}
$$

for $1 \leq p, q, r \leq \infty$ such that $1 / p+1 / q=1+1 / r\left(\|\cdot\|_{p}\right.$ is the norm in $\left.L^{p}\right)$. 
Let us denote by $g$ the function

$$
\mathbb{R}_{+} \ni s \longmapsto g(s)=e^{-s A_{+}} \in \mathcal{L}(E) .
$$

Then

$$
\int_{0}^{t} e^{-(t-s) A_{+}} f_{+}(s) d s=\left(g * f_{+}\right)(t) .
$$

Taking in the Young inequality $r=2, q=2, p=1$, we have the estimate

$$
\begin{aligned}
&\left\|g * f_{+}\right\|_{2} \leq\|g\|_{1} \cdot\left\|f_{+}\right\|_{2}=\int_{0}^{\infty}\left\|e^{-s A_{+}}\right\| d s \cdot\left(\int_{0}^{\infty}\left\|f_{+}\right\|^{2} d s\right)^{1 / 2} \\
& \quad \leq \int_{0}^{\infty} M e^{-s \alpha} d s \cdot\left(\int_{0}^{\infty}\left\|f_{+}(s)\right\|^{2} d s\right)^{1 / 2}<\infty
\end{aligned}
$$

It follows that the function

$$
\overline{\mathbb{R}}_{+} \ni t \longmapsto \int_{0}^{t} e^{-(t-s) A_{+}} f_{+}(s) d s \in X_{+}
$$

belongs to $L^{2}$, and therefore the function (3) belongs to $L^{2}$. Because $\dot{u}_{+}=f_{+}-A_{+} u_{+} \in L^{2}$ we deduce that $u_{+} \in H^{1}$.

The second problem of (2) is not an initial value problem but we will show that it has a unique solution in the class $H^{1}$. Any solution of

$$
\dot{u}_{-}+A_{-} u_{-}=f_{-}
$$

is given by the formula

$$
u_{-}(t)=e^{-t A_{-}} u_{-}(0)+\int_{0}^{t} e^{-(t-s) A_{-}} f_{-}(s) d s=e^{-t A_{-}}\left(u_{-}(0)+\int_{0}^{t} e^{s A_{-}} f_{-}(s) d s\right) .
$$

We observe first that the limit

$$
\lim _{t \rightarrow \infty} \int_{0}^{t} e^{s A_{-}} f_{-}(s) d s=\int_{0}^{\infty} e^{s A_{-}} f_{-}(s) d s \in X_{-}
$$

exists (this follows from the fact that $\sigma\left(A_{-}\right) \subset \mathbb{C}_{-}$).

Next, let us notice that

$$
u_{-}(0)+\int_{0}^{\infty} e^{s A_{-}} f_{-}(s) d s=0 .
$$

Indeed, if the above does not hold then the norm of the expression

$$
e^{-t A_{-}}\left(u_{-}(0)+\int_{0}^{t} e^{s A_{-}} f_{-}(s) d s\right)
$$

tends to the infinity and the function $u_{-}$is not in $L^{2}$. Consequently $u_{-}$is not in $H^{1}$. Therefore, in order to have $u_{-} \in H^{1}$ the condition

$$
u_{-}(0)=-\int_{0}^{\infty} e^{s A_{-}} f_{-}(s) d s
$$

has to be satisfied. We can write the solution of (5) in the form

$$
u_{-}(t)=e^{-t A_{-}}\left(u_{-}(0)+\int_{0}^{\infty} e^{s A_{-}} f_{-}(s) d s-\int_{t}^{\infty} e^{s A_{-}} f_{-}(s) d s\right)
$$


and if we allow (6) it will take the form

$$
u_{-}(t)=-\int_{t}^{\infty} e^{(s-t) A} f_{-}(s) d s .
$$

Using again the Young inequality and the fact that $\sigma\left(A_{-}\right) \subset \mathbb{C}_{-}$we prove that $u_{-} \in L^{2}$ and in consequence $u_{-} \in H^{1}$. Then the condition (6) is also sufficient to have $u_{-} \in H^{1}$. Therefore the solutions of both problems in (2) are uniquely determined in $H^{1}$.

We have actually proved

Corollary 1. The semi-Cauchy problem

$$
\left\{\begin{array}{l}
\dot{u}+A u=f \\
u_{+}(0)=\zeta_{+} ; \quad \zeta_{+} \in X_{+}, f \in L^{2}
\end{array}\right.
$$

has a unique solution $u \in H^{1}$.

We are going to investigate the above problem if the pair of spaces $\left(X_{+}, X_{-}\right)$is perturbed via maps which are compact perturbations of the identity. For this purpose we introduce the following

Definition 1. Let $\left(X_{1}, X_{2}\right),\left(Y_{1}, Y_{2}\right)$ be two pairs of closed subspaces of the space $E$ such that $E=X_{1} \oplus X_{2}=Y_{1} \oplus Y_{2}$. We say that the pairs $\left(X_{1}, X_{2}\right)$ and $\left(Y_{1}, Y_{2}\right)$ are equivalent if there exist a compact mapping $B \in \mathcal{L}_{c}(E)$ and a finite-dimensional subspace $V$ of $E$ such that the mapping $I+B \in \mathcal{L}(E)$ is an isomorphism and one of the following conditions (a) or (b) is satisfied

$$
\begin{aligned}
& \text { (a) } \widehat{X}_{1}=Y_{1} \oplus V \text { and } \widehat{X}_{2} \oplus V=Y_{2} \\
& \text { (b) } \widehat{X}_{1} \oplus V=Y_{1} \text { and } \widehat{X}_{2}=Y_{2} \oplus V,
\end{aligned}
$$

where $\widehat{X}_{i}=(I+B)\left(X_{i}\right), i=1,2$.

PROPOSITION 2. The above relation is an equivalence relation.

The proof is elementary, but it needs some calculations. We omit it.

REMARK. If $E=\mathbb{R}^{N}$ then any two splittings $\left(X_{1}, X_{2}\right)$ and $\left(Y_{1}, Y_{2}\right)$ are equivalent in the above sense.

In what follows we will consider two decompositions of $E, X_{+} \oplus X_{-}=X_{1} \oplus X_{2}$. The first one is associated with the operator $A \in \mathcal{L}(E)$ satisfying $\sigma(A) \cap \mathbb{R} i=\emptyset$ and the second decomposition $E=X_{1} \oplus X_{2}$ is such that the pairs $\left(X_{1}, X_{2}\right),\left(X_{+}, X_{-}\right)$are equivalent in the above sense. Choose a compact operator $B \in \mathcal{L}_{c}(E)$ and a finite-dimensional subspace $V \subset E$ such that $I+B$ is an isomorphism and one of the following conditions holds

$$
\begin{array}{ll}
\text { (a) } \widehat{X}_{+}=X_{1} \oplus V \text { and } \widehat{X}_{-} \oplus V=X_{2} \\
\text { (b) } \widehat{X}_{+} \oplus V=X_{1} \text { and } \widehat{X}_{-}=X_{2} \oplus V,
\end{array}
$$

where as earlier $\widehat{X}_{ \pm}=(I+B)\left(X_{ \pm}\right)$.

Let $X, Y$ be closed subspaces of $E$ such that $E=X \oplus Y$. By $P_{X}: E \longrightarrow E$ we will denote the projection onto $X$ along $Y$.

Lemma 1. The operators $P_{X_{+}}-P_{\widehat{X}_{+}}, P_{X_{-}}-P_{\widehat{X}_{-}}, P_{\widehat{X}_{+}}-P_{X_{1}}, P_{\widehat{X}_{-}}-P_{X_{2}}$ are compact. 
Proof. We have of course $E=\widehat{X}_{+} \oplus \widehat{X}_{-}$. Then for each $w=z+B z \in E$ we have $w=P_{\widehat{X}_{+}} w+P_{\widehat{X}_{-}} w=P_{\widehat{X}_{+}}(z+B z)+P_{\widehat{X}_{-}}(z+B z)=\left(P_{\widehat{X}_{+}} z+P_{\widehat{X}_{+}} B z\right)+\left(P_{\widehat{X}_{-}} z+\right.$ $\left.P_{\widehat{X}_{-}} B z\right)=w_{+}+w_{-} \in \widehat{X}_{+} \oplus \widehat{X}_{-}$.

On the other hand, $w=z+B z=P_{X_{+}} z+P_{X_{-}} z+B\left(P_{X_{+}} z+P_{X_{-}} z\right)=P_{X_{+}} z+$ $B P_{X_{+}} z+P_{X_{-}} z+B P_{X_{-}} z=(I+B) P_{X_{+}} z+(I+B) P_{X_{-}} z=\widehat{w}_{+}+\widehat{w}_{-} \in \widehat{X}_{+} \oplus \widehat{X}_{-}$. From the uniqueness of the decomposition we get $w_{+}=\widehat{w}_{+}$and $w_{-}=\widehat{w}_{-}$. Therefore $P_{\widehat{X}_{+}} z+P_{\widehat{X}_{+}} B z=P_{X_{+}} z+B P_{X_{+}} z$ and $P_{X_{+}}-P_{\widehat{X}_{+}}=P_{\widehat{X}_{+}} B-B P_{X_{+}} \in \mathcal{L}_{c}(E)$. Similarly $P_{\widehat{X}_{-}} z+P_{\widehat{X}_{-}} B z=P_{X_{-}} z+B P_{X_{-}} z$ and $P_{X_{-}}-P_{\widehat{X}_{-}}=P_{\widehat{X}_{-}} B-B P_{X_{-}} \in \mathcal{L}_{c}(E)$.

Now let condition (a) from (8) be fulfilled. Then $E=X_{1} \oplus \widehat{X}_{-} \oplus V$. For any $w \in E$ we have $w=P_{\widehat{X}_{+}} w+P_{\widehat{X}_{-}} w \in \widehat{X}_{+} \oplus \widehat{X}_{-}$and $w=P_{X_{1}} w+P_{X_{2}} w=P_{X_{1}} w+P_{\widehat{X}_{-}} P_{X_{2}} w+$ $P_{V} P_{X_{2}} w=\left(P_{X_{1}} w+P_{V} P_{X_{2}} w\right)+P_{\widehat{X}_{-}} P_{X_{2}} w \in \widehat{X}_{+} \oplus \widehat{X}_{-}$. It follows that $P_{\widehat{X}_{+}} w=P_{X_{1}} w+$ $P_{V} P_{X_{2}} w$. Therefore $P_{\widehat{X}_{+}}-P_{X_{1}}=P_{V} P_{X_{2}} \in \mathcal{L}_{c}(E)$ (since $P_{V}$ is finite-dimensional). Similarly $w=P_{\widehat{X}_{+}} w+P_{\widehat{X}_{-}} w=P_{X_{1}} P_{\widehat{X}_{+}} w+\left(P_{V} P_{\widehat{X}_{+}} w+P_{X_{2}} w\right) \in X_{1} \oplus X_{2}$ and $w=$ $P_{X_{1}} w+P_{X_{2}} w \in X_{1}^{+} \oplus X_{2}$. Hence $P_{V} P_{\widehat{X}_{+}} w+P_{\widehat{X}_{-}} w=P_{X_{2}} w$ and $P_{\widehat{X}_{-}}-P_{X_{2}}=-P_{V} P_{\widehat{X}_{+}} \in$ $\mathcal{L}_{c}(E)$.

If condition (b) from (8) is fulfilled the proof is similar.

Theorem 1. Let $A \in \mathcal{L}(E), \sigma(A) \cap \mathbb{R} i=\emptyset$ and $\left(X_{+}, X_{-}\right),\left(X_{1}, X_{2}\right)$ be equivalent pairs. Then the continuous linear mapping $T: H^{1} \longrightarrow L^{2} \times X_{1}$ defined by

$$
T u:=\left(\dot{u}+A u, u_{1}(0)\right)
$$

is a Fredholm map of index

$$
\operatorname{ind}(T)= \begin{cases}\operatorname{dim} V & \text { if } \widehat{X}_{+}=X_{1} \oplus V \text { and } \widehat{X}_{-} \oplus V=X_{2} \\ -\operatorname{dim} V & \text { if } \widehat{X}_{+} \oplus V=X_{1} \text { and } \widehat{X}_{-}=X_{2} \oplus V .\end{cases}
$$

Proof. Assume condition (a) holds, i.e. $\widehat{X}_{+}=X_{1} \oplus V$ and $\widehat{X}_{-} \oplus V=X_{2}$.

Then $E=X_{1} \oplus V \oplus \widehat{X}_{-}$. The map

$$
H^{1} \ni u \stackrel{\widehat{T}}{\longrightarrow}\left(\dot{u}+A u,(I+B) u_{+}(0)\right) \in L^{2} \times \widehat{X}_{+}
$$

is a superposition of isomorphisms

$$
H^{1} \ni u \stackrel{T_{+}}{\longrightarrow}\left(\dot{u}+A u, u_{+}(0)\right) \stackrel{(I, I+B)}{\longrightarrow}\left(\dot{u}+A u,(I+B) u_{+}(0)\right) \in L^{2} \times \widehat{X}_{+},
$$

where $T_{+}(u):=\left(\dot{u}+A u, u_{+}(0)\right)$ is the isomorphism given by Proposition 1 . Let $\pi: L^{2} \times$ $\left(X_{1} \oplus V\right) \longrightarrow L^{2} \times X_{1}$ be the epimorphism given by the formula $\pi\left(f, \widehat{\zeta}_{+}\right)=\left(f, P_{X_{1}}\left(\widehat{\zeta}_{+}\right)\right)$, where $P_{X_{1}}: X_{1} \oplus V \longrightarrow X_{1}$ is the projection operator and let $T_{1}:=\pi \circ \widehat{T}$. Then $T_{1}$ is the epimorphism which can be written by the formula

$$
H^{1} \ni u \longmapsto T_{1} u=\left(\dot{u}+A u, P_{X_{1}}(I+B) u_{+}(0)\right) \in L^{2} \times X_{1} .
$$


We illustrate all above mappings in the following diagram

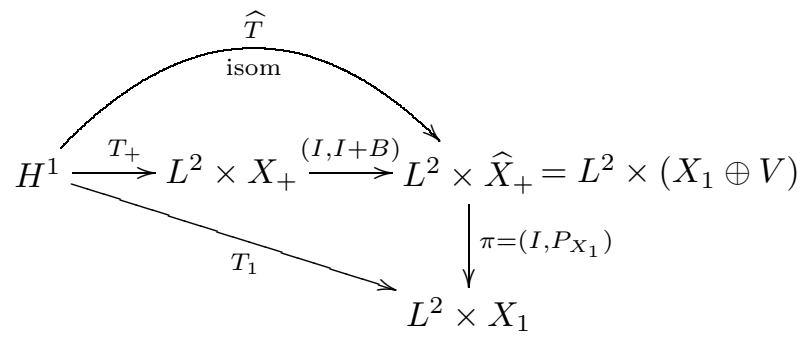

Directly from the definition of the Fredholm index one easily obtains

$$
\operatorname{ind}\left(T_{1}\right)=\operatorname{dim} \operatorname{Ker}\left(T_{1}\right)-\operatorname{codim} \operatorname{Im}\left(T_{1}\right)=\operatorname{dim} V .
$$

We will show that $\operatorname{ind}\left(T_{1}\right)=\operatorname{ind}(T)$. It is enough to show that $T_{1}-T \in \mathcal{L}_{c}(E)$. Indeed,

$$
\begin{aligned}
\left(T_{1}-T\right)(u)=(0, & \left.(I+B) u_{+}(0)-u_{1}(0)\right) \\
& =\left(0,(I+B) P_{X_{+}} u(0)-P_{X_{1}} u(0)\right)=\left(0,\left(P_{X_{+}}-P_{X_{1}}+B P_{X_{+}}\right) u(0)\right) .
\end{aligned}
$$

Since $P_{X_{+}}-P_{X_{1}}=P_{X_{+}}-P_{\widehat{X}_{+}}+P_{\widehat{X}_{+}}-P_{X_{1}}$ and $P_{X_{+}}-P_{\widehat{X}_{+}}, P_{\widehat{X}_{+}}-P_{X_{1}}, B P_{X_{+}}$are compact, the map $T_{1}-T \in \mathcal{L}_{c}(E)$.

Now let condition (b): $\widehat{X}_{+} \oplus V=X_{1}$ and $\widehat{X}_{-}=X_{2} \oplus V$ be satisfied. Then $E=$ $\widehat{X}_{+} \oplus V \oplus X_{2}$. Let $\iota: L^{2} \times \widehat{X}_{+} \hookrightarrow L^{2} \times\left(\widehat{X}_{+} \oplus V\right)=L^{2} \times X_{1}$ be the embedding map and let $T_{2}:=\iota \circ \widehat{T}$,

$$
H^{1} \ni u \longmapsto T_{2} u=\left(\dot{u}+A u,(I+B) u_{+}(0)\right) \in L^{2} \times X_{1} .
$$

Consider the following diagram

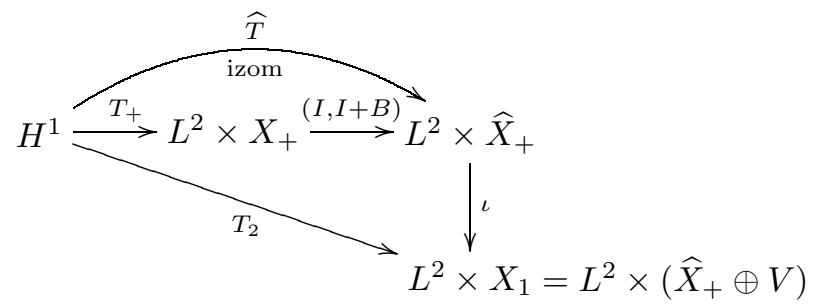

The map $T_{2}$ is a Fredholm operator of $\operatorname{ind}\left(T_{2}\right)=-\operatorname{dim} V\left(\operatorname{dim} \operatorname{Ker}\left(T_{2}\right)=0\right.$ and $\left.\operatorname{codim} \operatorname{Im}\left(T_{2}\right)=\operatorname{dim} V\right)$. As earlier it can be shown that $T_{2}-T \in \mathcal{L}_{c}(E)$ and it follows that $\operatorname{ind}\left(T_{2}\right)=\operatorname{ind}(T)$.

We can reformulate the above theorem to obtain the following result.

COROLlary 2. If $T$ from Theorem 1 is an isomorphism then the space of solutions of the problem

$$
\left\{\begin{array}{l}
\dot{u}+A u=f \\
u_{1}(0)=\zeta_{1} ; \quad \zeta_{1} \in X_{1}, f \in L^{2}
\end{array}\right.
$$

is of dimension ind $(T)$. In particular, if $\operatorname{ind}(T)=0$ then this problem has a unique solution in $H^{1}$. 
The nonlinear case of (1) will be discussed in the next paper.

\section{References}

[1] R. A. Adams, Sobolev Spaces, Academic Press, New York 1975.

[2] T. Kato, Perturbation Theory for Linear Operators, Springer, Berlin 1980.

[3] P. J. Rabier, C. A. Stuart, A Sobolev space approach to boundary value problem on the half-line, Commun. Contemp. Math. 7 (2005), 1-36.

[4] S. Secchi, C. A. Stuart, Global bifurcation of homoclinic solutions of Hamiltonian systems, Discrete Contin. Dyn. Syst. 9 (2003), 1493-1518. 
